\title{
Synthesis and morphologic characterization of hollow gold nanoparticles with different amount of gold and its possible use as radio sensitizer
}

\author{
J. Medina-Monares ${ }^{1}$, E. Ortiz-Rascón ${ }^{2}$, L. Baez-Castillo ${ }^{1}$, R. Carrillo-Torres ${ }^{1}$ and M.E. Álvarez-Ramos ${ }^{1}$ \\ 1. Departamento de Física, Universidad de Sonora (UNISON), Blvd. Luis Encinas y Rosales s/n, Col. \\ Centro, Hermosillo, Sonora, CP. 83000, México. \\ 2. CONACYT - Departamento de Física, Universidad de Sonora (UNISON), Blvd. Luis Encinas y \\ Rosales s/n, Col. Centro, Hermosillo, Sonora, CP. 83000, México.
}

We present results obtained by synthesis and morphologic characterization of silver and gold hollow nanoparticles synthetized by galvanic replacement method [1] and its possible use as a modifier of absorbed energy (increase radio sensitivity of local media) [2]. These hollow nanoparticles will be used as a radio sensitizer due to their high atomic number. Recent studies have reported an increment in radio sensibility on materials with biologic relevance by adding gold nanoparticles in such materials [4]. Some authors associate such growth to the energy of the photons used during the irradiation; also, they associate this effect with the size of the nanoparticles used. However, some of these results are not conclusive enough. It is believed that this increase is related to the local increase in the atomic number which is directly associated with an increase in the effect of pair production on the material.

The change in the absorbed dose (energy per unit of mass) can be quantified by using an appropriate dosimeter in a media containing the nanoparticles solution, presented in Fig. 1, used as a buildup region. This array can be irradiated with some different energies. In this work we show that the size and the shape of the hollow nanoparticles remains unaltered independently of the different amount of tetrachloroauric acid used. In Fig. 1 we show the different nanoparticles solutions vials (left) with an increasing volume of tetrachloroauric acid by $20 \mu \mathrm{l}$ steps from left to right; in Fig. 1 we can also appreciate the plasmon displacement for these different gold concentrations in accordance with previous reported work [1].

For the two volume concentrations of tetrachloroauric acid: 20 and $100 \mu 1$ in Fig. 2 we show the SEM images for different magnifications, these images were obtained by using a scanning electron microscope (SEM) model FE-SEM JEOL JSM-7800F. As the images suggest, the size and the shape of these nanoparticles are independent of the volume concentration of tetrachloroauric acid used to synthesize them. In this way, the main variable in these nanoparticles is the amount of gold within the shells. As the literature suggest, it is expected a variation on the dose registered by a suitable dosimeter containing these nanoparticles; further work needs to be done in this direction.

References:

[1] R C Carrillo-Torres et al, RSC Advances, 6(47) (2016), p. 41304-41312.

[2] A Alkhatib et al, Medical Physics, 36(8), (2009), p. 3543-8.

[3] J F Hainfeld et al, Physics in Medicine and Biology, 49(18). (2004), p. 309-15.

[4] A Mesbahia. Reports of Practical Oncology and Radiotherapy, 15(6). (2010) p. 176-80.

[5] The authors acknowledge funding from the Consejo Nacional de Ciencia y Tecnología (CONACyT), through project 255791-INFR 2015. 

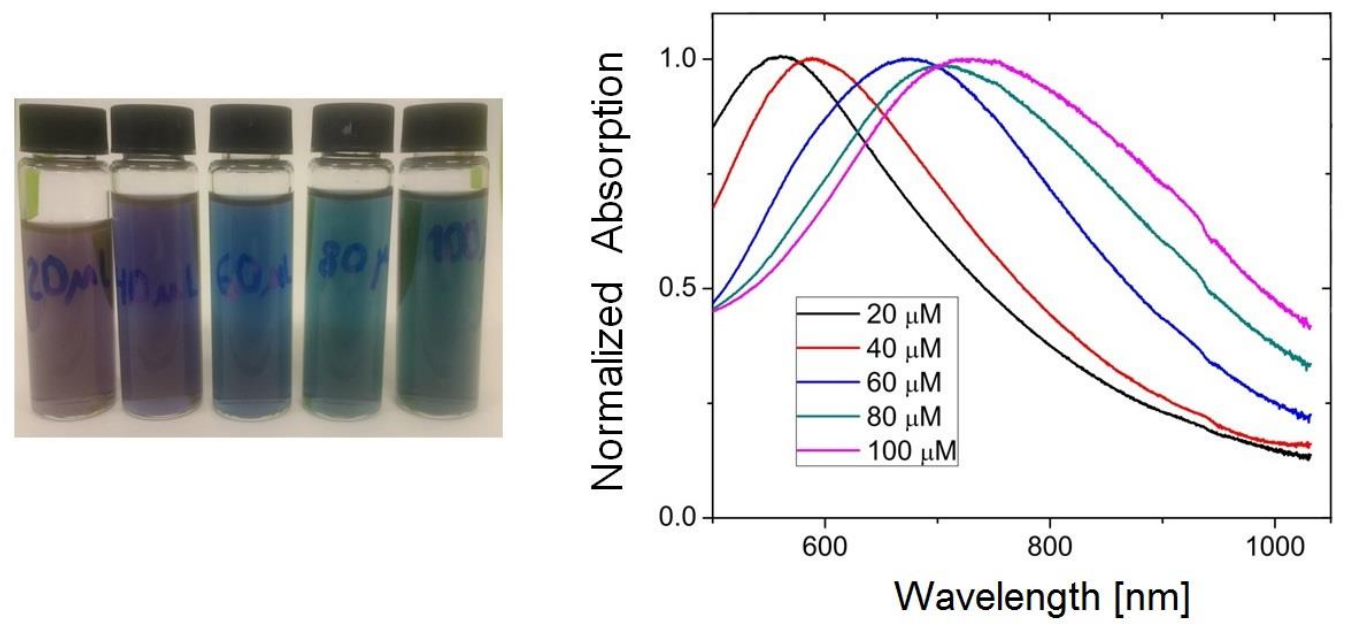

Figure 1. The different nanoparticles suspensions vials (left) with an increasing volume of tetrachloroauric acid by $20 \mu \mathrm{l}$ steps from left to right. On the right we can appreciate the plasmon displacement for these different gold concentrations.
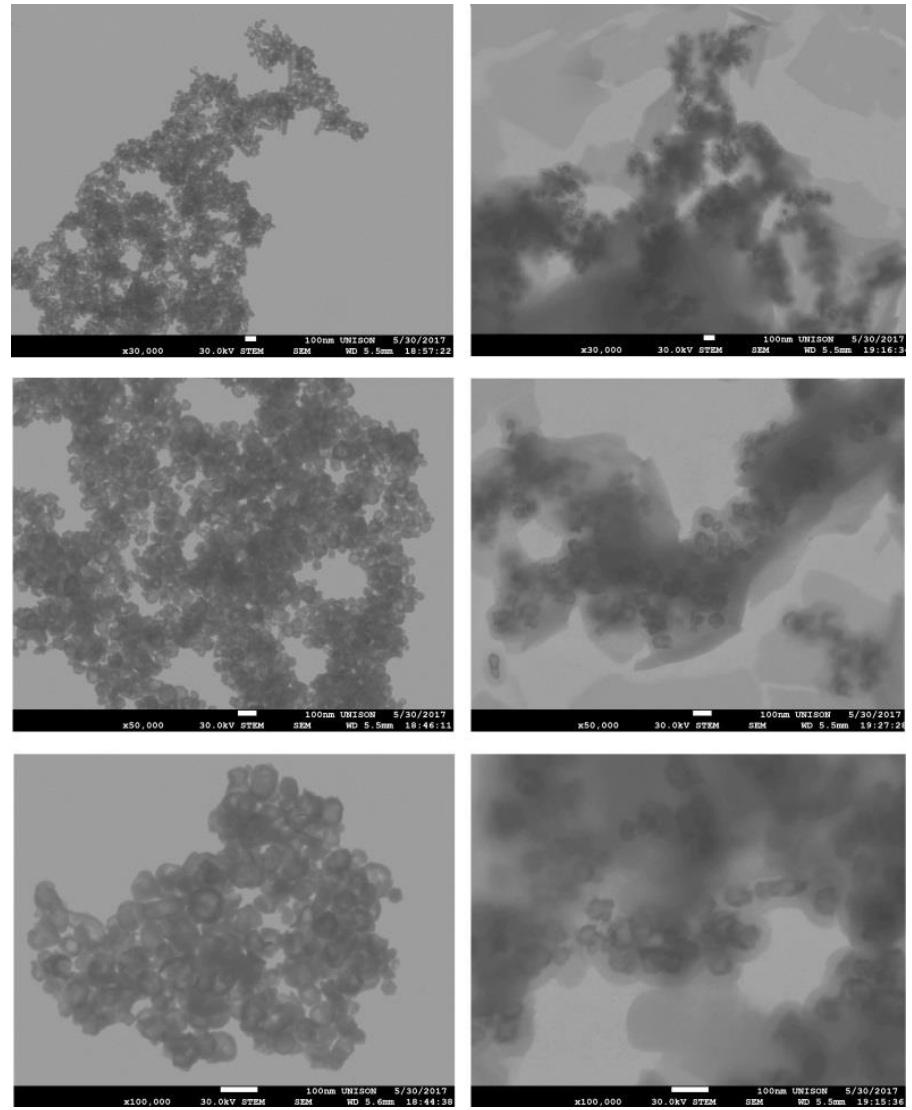

Figure 2. For two volume concentrations 20 and $100 \mu$ (left and right columns, respectively) we show the SEM images for different magnifications. As the images suggest, the size and the shape of the nanoparticles is independent on the volume concentration of tetrachloroauric acid used to synthesize them. 